\title{
Bend and survive
}

Victor Steinberg

The aim of aerodynamic design is to reduce the drag experienced by a body, such as a car, in a flowing medium, such as air. But what happens if the body is flexible and bends in response to the flow?

E veryone has seen trees, particularly young ones, bend in a strong wind. But fewer people realize that this is a clever way of reducing the drag force of the wind on the tree. Thus trees combine a functional necessity to grow tall and carry out photosynthesis with the ability to survive strong winds (Fig. 1). But just how much can a flexible object reduce drag by changing its shape? On page 479 of this issue, Alben, Shelley and Zhang ${ }^{1}$ present results from their experimental and theoretical studies of drag reduction by shape reconfiguration for a flexible body immersed in a flowing medium.

When the velocity of a flowing medium increases, there is always an accompanying increase in the resistance of a body to the flow $^{2}$. The effect is even greater if the flow velocity exceeds some critical value and becomes turbulent, which is often the case in natural phenomena and engineering applications. The most effective way to counteract flow resistance is to design a body whose shape minimizes drag by reducing, or delaying, the turbulence in the boundary layer of the flow nearest the moving body. This is reflected in the design of aerodynamic shapes - from planes and trains, to cars and cyclists' helmets.

But these examples are all bodies with a fixed shape. A more ingenious way to reduce

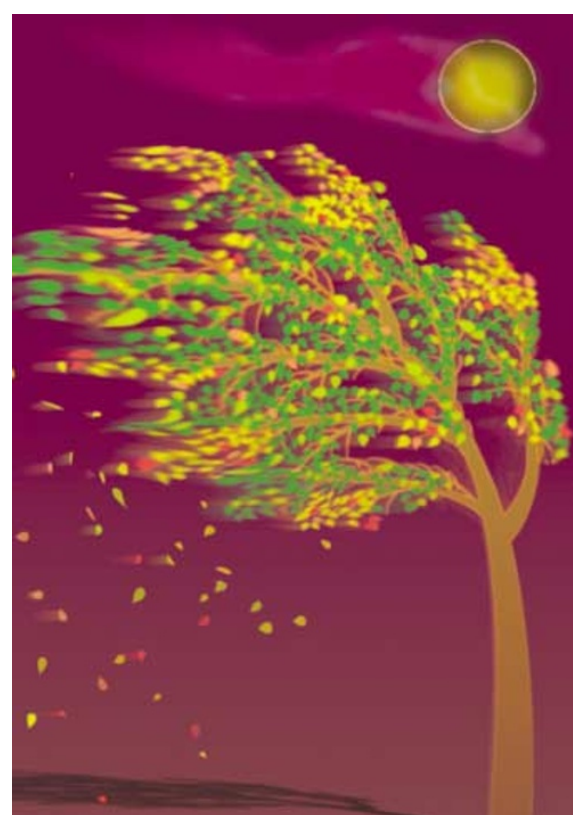

Figure $1 \mathrm{~A}$ tree bends in a gusty wind, reducing the potentially damaging drag force of the moving air on its branches. drag, as seen in nature - from trees to jellyfish to amoebae - is for an immersed body to adjust its shape as the flow velocity increases. The only engineering application of this effect that immediately comes to mind is a sail — although, in this case, the reshaping of the sail in a stiff wind actually increases the drag, and hence drives the boat.

The outstanding feature of the studies presented by Alben et al. ${ }^{1}$ is that they have designed an experiment that can be closely described by a theoretical model. The authors studied the behaviour of a twodimensional flexible fibre immersed in flowing soapy water. The soap film makes the forces on the fibre easily visible (see Fig. 2 on page 480), and the flow velocity and the force on the fibre can be measured simultaneously. Using a model that couples the flow dynamics with the bending of the fibre, Alben et al. have derived a differential equation describing the curvature of the fibre in the flow that can be solved for any given value of a control parameter. This parameter is the nondimensional velocity, $\eta$, defined as the ratio of the fluid kinetic energy to the bending elastic energy of a flexible body.

The model predicts a sharp transition from a nearly straight fibre to a bending one when the flow kinetic energy becomes comparable to the fibre's elastic bending energy - that is, when the value of the control parameter is of order one. One of the consequences of this transition is a change in the functional dependence of the drag on $\eta$, an effect that Alben et al. also see experimentally.

The particularly striking feature of the transition shows up in the shape adopted by the bending fibre. The fibre takes on a quasiparabolic shape, and, if the overall length of the fibre (whatever its value) is scaled by a factor $\eta^{2 / 3}$, the parabola is always the same (Fig. 2). As $\eta$ increases, so the increased bending of the fibre is confined to a progressively smaller region at its ends; the size of this region is related to a new parameter in the problem, the 'bending length' of the fibre. This length parameter arises from the competition between the rigidity of the fibre and the force of the flow and sets the 'self-similarity' scale for the parabolic bending shape. For large values of $\eta$, the total drag on the fibre is created by the tip region defined by the bending length, and not by the reduced fibre-profile width as might be assumed.

There are limitations in the comparison

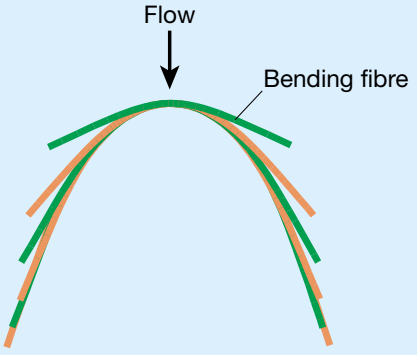

Figure 2 The curvature of a flexible fibre in a flowing medium increases with the strength of the flow. Alben et al. ${ }^{1}$ show that, in fact, the curvature increases only around the tips of the fibre, and the central region maintains a quasiparabolic, self-similar shape.

between the experiment and the model, some of which Alben et al. acknowledge in their paper. For example, the compressibility of the soap film, a result of thickness variations and skin friction, can alter the scaling behaviour. Another factor that is not mentioned is the surface tension between the soap film and the fibre. These effects probably account for the almost uniform systematic factor that Alben et al. have to apply to achieve a good quantitative comparison between the theory and their data.

There is certainly more to be learned from further studies of fibre bending, such as whether hysteresis (a difference between bending and unbending) occurs. It would also be interesting to extend the experiment to three dimensions, using a flexible body made of a gel with an appropriate elastic modulus, immersed in a three-dimensional flow. Whether the transition to bending and the self-similar shape survive in higher dimensions could then be verified.

Victor Steinberg is in the Department of Physics of Complex Systems, Weizmann Institute of Science, Rehovot 76100, Israel.

e-mail:victor.steinberg@weizmann.ac.il

1. Alben, S., Shelley, M. \& Zhang, J. Nature 420, 479-481 (2002). 2. Landau, L. D. \& Lifshitz, E. M. Fluid Mechanics (Pergamon, Oxford, 1987)

\section{correction}

In Hans-Georg Rammensee's article "Immunology: Survival of the fitters" (Nature 419, 443-445; 2002), it was said that MHC class I ligands (peptide fragments that convey information to the immune system) are made up of exactly nine amino acids. This was a simplification, in that all copies of a particular MHC class I ligand are of a uniform length, and most are exactly nine amino acids long. Some, however, are composed of eight, ten or eleven amino acids, and there may be other exceptions. 